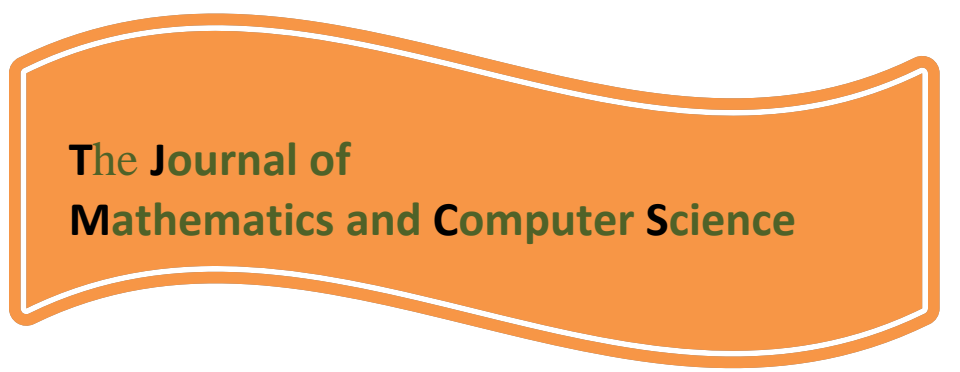

Available online at

http://www.TJMCS.com

The Journal of Mathematics and Computer Science Vol .1 No.4 (2010) 339-348

\title{
Fuzzy Logic Applications in Chemical Processes
}

\author{
M. R. Sarmasti Emami \\ Iran University of Science \&Technology \\ m_r_emami@iust.ac.ir
}

Received: June 2010, Revised: October 2010

Online Publication: December 2010

\begin{abstract}
:
Fuzzy logic a new approach was intended to emulate human reasoning using calculations and operations with fuzzy groups and linguistic variables. Fuzzy variables describe qualitative expressions such as very slow, slow, fast, very fast, and so on. The application of fuzzy logic techniques has been increasing rapidly in the last few years. Fuzzy logic is used in target tracking, pattern recognition, robotics, power systems, controller design, chemical engineering, biomedical engineering, vehicular technology, economy management and decision making, aerospace applications, communications and networking, electronic engineering, and civil engineering. In many chemical engineering systems, the classification of product quality characteristics is performed by human experts, due to the absence of measuring devices. The development of mathematical models for such systems is a rather difficult task, since no equations based on first principles can be written. Chemical engineering has employed fuzzy logic in the detection of chemical agents as well as gas recognition. It has also been applied to processes control, batch distillation column, separation process, and kinetics. In this research, we investigate these applications in more detail.
\end{abstract}

Keywords: Chemical, Process, Fuzzy Logic, System

\section{Introduction}

Chemical engineering is the branch of engineering that deals with the application of physical science (e.g., chemistry and physics), and life sciences (e.g., biology, microbiology and biochemistry) with mathematics, to the process of converting raw materials or chemicals into more useful or 
valuable forms. In addition to producing useful materials, modern chemical engineering is also concerned with pioneering valuable new materials and techniques - such as nanotechnology, fuel cells and biomedical engineering. Chemical engineering largely involves the design, improvement and maintenance of processes involving chemical or biological transformations for large-scale manufacture. Chemical engineers ensure the processes are operated safely, sustainably and economically. Chemical processes include manufacturing phases such as, filling or emptying a reactor, heating or mixing a product. Intelligent control is a control system with the ultimate degree of autonomy in terms of self-learning, self-reconfigurability, reasoning, planning and decision making, and the ability to extract the most valuable information from unstructured and noisy data from any dynamically complex system and/or environment [1]. Complex industrial processes such as a batch chemical reactors; blast furnaces, cement kilns and basic oxygen steel making are difficult to control automatically. This difficulty is due to their nonlinear; time varying behavior and the poor quality of available measurements. In such cases automatic control is applied to those subsidiary variables, which can be measured and controlled, for example temperatures, pressures and flows. The overall process controls objectives, such as the quality and quantity of product produced, has

been left in the hands of the human operators in the past [2].

In recent years, computational intelligence has been used to solve many complex problems by developing intelligent systems. Fuzzy logic has proved to be a powerful tool for decision-making systems, such as expert and pattern classification systems. Fuzzy set theory has been used in some chemical process. In traditional rule-based approaches, knowledge is encoded in the form of antecedent-consequent structure. When new data are encountered, it is matched to the antecedent's clause of each rule, and those rules where antecedents match a data exactly are fired, establishing the consequent clauses. This process continues until the desired conclusion is reached, or no new rule can be fired. In the past decade, fuzzy logic has proved to be useful for intelligent systems in chemical engineering. In this paper, we discuss how fuzzy set theory and fuzzy logic can be used to develop knowledge-based systems in chemical engineering. Also we focused on the applications of it in chemical processes.

\section{Fuzzy Set Theory and Fuzzy Logic System}

"As the complexity of a system increases, our ability to make precision and yet significant statements about the behavior diminishes until a threshold is reached beyond which precise and significance become almost mutually exclusive characteristics [3]. The concept of fuzziness was first proposed by [4]. He aimed to describe complex and complicated systems using fuzzy approximation and introduced fuzzy sets. "Generally, fuzzy logic can be considered as a logical system that provides a model for modes of human reasoning that are approximations rather than exact. Fuzzy logic systems had found successful applications in wide variety of fields such as: automatic control, pattern recognition, signal processing, expert systems, communication, system identification and time series prediction. In chemical engineering systems, they have been generally used in control studies. Since Fuzzy Logic Control (FLC) does not require a model and the control is based on expertise human reasoning, they have been applied in many control schemes. Also, they are used extensively in modeling. . Every fuzzy logic system is composed of rules, fuzzifier, inference engine and defuzzifier (Figure 1). 


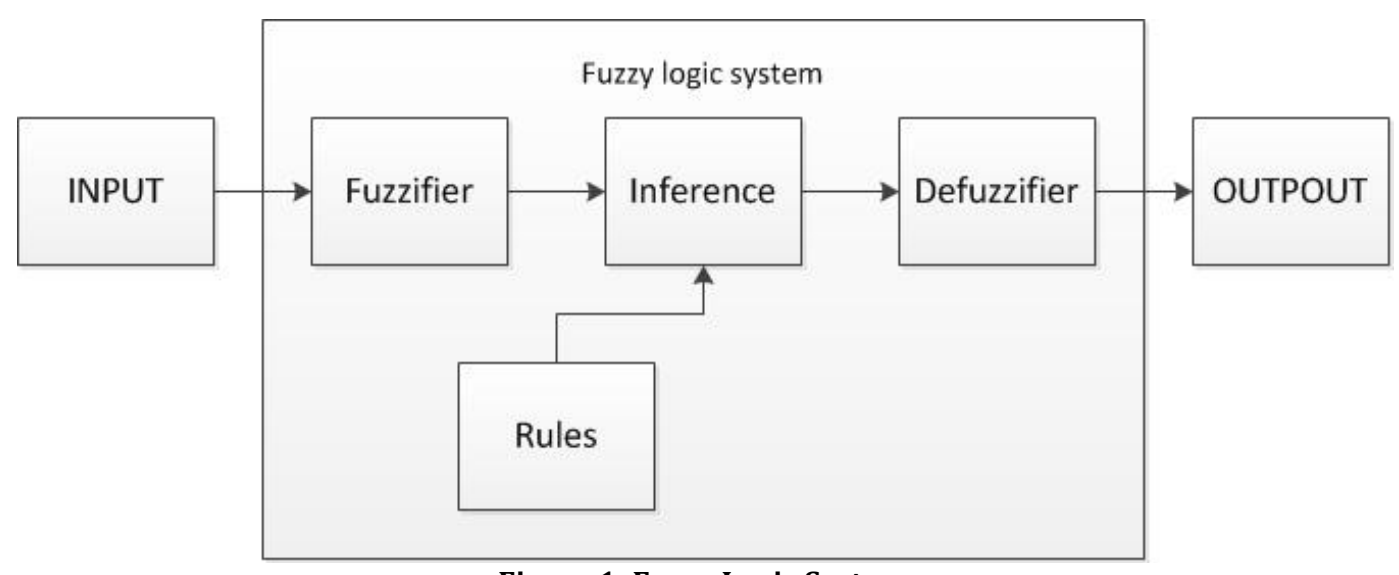

Figure 1: Fuzzy Logic System

.The advantages of fuzzy controllers based on fuzzy logic systems are intuitive design, reflecting the behavior of human operator, the fact that the model of the controlled process is not necessary (an important feature when ill-defined processes are to be controlled), and good control quality (not worse than that of classical controllers). However, the main disadvantages are the necessity of acquisition and preprocessing of the human operator's knowledge about the controlled process, sequential search through rule bases, and time consuming defuzzification methods.

\section{Applications of Fuzzy Logic System in Chemical Processes}

Chemical engineering has employed fuzzy logic in the detection of chemical agents as well as gas recognition. It has also been applied to processes control, batch distillation column, phosphoric acid production, nuclear research, separation process, controlling the power output of a nuclear reactor and kinetics. In this research, we investigate these applications in more detail.

\subsection{Piping risk assessment}

Pipelines are generally recognized to be the safest and most economical way of transporting hazardous substances in comparison with other methods of transport, for example by road and rail. However, the different databases of accidents reported a number of pipeline failures with a release of transported medium to the environment and substantial losses. Adam et al. explore the application of the fuzzy logic for risk assessment of major hazards connected with transportation of flammable substances in long pipelines [5].

\subsection{Safety analysis}

Process and chemical plants, where large amounts of dangerous chemical substances are stored and handled, may be subjected to different types of hazards including natural hazards, process hazards as well as terrorist and criminal acts. A successful management of such facilities reuires pertinent information and good judgment about the hazards posed by the activity of that facility. Such exercises called Process Safety Analyses (PSA) enable decisions concerning the selection of appropriate technical and organizational safety measures in order to manage the identified risk and to meet risk acceptance criteria as are required in some European countries.

Process Safety Analysis (PSA), being a basis for decision-making process in chemical industry is a very complex task, representing a number of uncertainties connected with information shortages which may lead to the important overlooking of the safety assurance of plants. 2 . The "bow-tie" models consisting of the fault tree and event tree for a particular accident scenario are knowledge-acquisition structures and therefore they require special treatment of subjective 
uncertainty. The application of fuzzy sets may improve data acquisition process. of course, The success of this method depends on quality of failure data collection of process components as well as on the cooperation with plant operation staff [6].

Efforts to provide work safety in workplaces, such as risk management, are not only important for the health of workers but also inevitable managerial activities for economic and financial performance, productivity of the facility and the quality and continuity of production [7]. Because of the hazardous nature of construction work, occupational safety is a serious problem in the construction industry. The nature of construction work ensures that uncertainties are inherent in every condition; and on-site inspections generally use linguistic expressions rather than metrics to assess the risks of workers at a construction site. Additionally legal records, statistical data and documentation produced by companies are generally insufficient for determining risk.

Gurcanli and Mungen proposed a method for assessment of the risks that workers expose to at construction sites using a fuzzy rule-based safety analysis to deal with uncertain and insufficient data. Using this approach, historical accident data, subjective judgements of experts and the current safety level of a construction site can be combined. In the scope of this study, first 5239 occupational accidents in the construction industry are identified from 40,000 unclassified occupational accidents in all industries [8].The method is then implemented on a tunneling construction site and risk level for all type of accidents is derived

\subsection{Furnace control}

Many furnaces have very long time constants and no single variable that can be used to control the process. For these furnaces rule-based expert systems and fuzzy logic control systems are commonly used. These systems use inputs from a wide range of measurable furnace parameters, such as fuel input, exit gas temperature, exit gas oxygen, exit gas $\mathrm{CO}$, exit gas $\mathrm{NO}_{\mathrm{x}}$, feed rate, firing rate, feed/product residence time, etc., to infer the furnace operating conditions. The control system then adjusts the furnace inputs to obtain a set of parameters most conducive to optimum product quality and production rate. These systems effectively mimic the best human operators but do so with greater consistency than real operators are able to maintain. Owing to the complexity of the processes involved, these systems are expensive to install commission and tune. However, the increased production, improved product quality and reduced specific energy consumption ensures that the return on investment is very good [9].

\subsection{Modeling of the Fluidized Catalytic Cracking Unit of a Petrochemical Refinery}

Fluid catalytic cracking (FCC) is an important oil refinery process, which converts high molecular weight oils into lighter hydrocarbon products. About $45 \%$ of worldwide gasoline production comes from FCC processes and its ancillary units. Optimization of gasoline production poses a big challenge in the petrochemical refinery because the input and output variables are nonlinear, interdependent, and full of uncertainties. FCC processes are known to be very difficult to model and control because of the large process scale, complicated hydro-dynamics and complex kinetics of both cracking and coke burning reactions.

Osofisan and Obafaiye describe investigations carried out regarding the application of Fuzzy Logic Control to the Fluidized Catalytic Cracking Unit (FCCU) of Kaduna Refinery and Petrochemical Company in Northern Nigeria, as a case study [10]. This research has demonstrated and established the advantage of relative ease of design and implementation of control systems offered by Fuzzy Logic. Also, Taskin et al. used to the fuzzy logic for control of the FCC unit. They indicated how fuzzy 
logic control (FLC), as a promising controls technique, would be effectively used for improved process control of FCC in refinery process industry [11].

\subsection{Classification of product qualitative}

There are many chemical engineering processes, where the quality characteristics of the product cannot be measured objectively either on-line due to the lack of proper sensors or off-line due to the absence of any measuring devices. In these cases, a human expert is employed to assign the product quality characteristics to certain predefined categories (classes), based on his experience and perceptions. The procedure of employing a human expert to perform the classification usually requires the interruption of the process in order to collect a sample. Furthermore, this way of classifying the product quality is very subjective and may lead to significant errors, especially when the same expert is not always employed to perform the classification.

A different approach to classify quality parameters is presented here, based on the fuzzy logic theory. This approach uses a fuzzy logic technique to develop models, where quality variables are inferred from other accurately measured process variables. Tsekouras et al. proposed a fuzzy classifier, which can be used as an adequate and reliable expert system to perform quality classifications. The methodology performs remarkably well in two different cases, showing that it has certain advantages over other black-box modeling techniques [12].

\subsection{Separation Process}

In chemistry and chemical engineering, a separation process is used to transform a mixture of substances into two or more distinct products. The separated products could differ in chemical properties or some physical property, such as size, or crystal modification or other separation into different components.

\subsubsection{Gas Stream}

Recovery of heavier hydrocarbons such as LPG, LNG and volatile organic compounds (VOCs) from gas streams is of great importance economically. When the rates of these gas streams are modest and heavier hydrocarbons are the minor components of these gas streams, employing membrane gas separation process with rubbery membranes such as poly dimethyl siloxane (PDMS) becomes rational. Ghadimi et al. proposed a novel model was developed to predict separation performance of different membranes. The developed model is able to choose the best conditions of preparation for the next membranes heuristically. The model can be easily applied to reduce membrane preparation cost and time, for all types of membranes [13].

\subsubsection{Oil Stream}

The basic separation process performed by the oil battery is described as follows, and a typical schematic flow diagram for an oil battery is shown in Figure 2. 


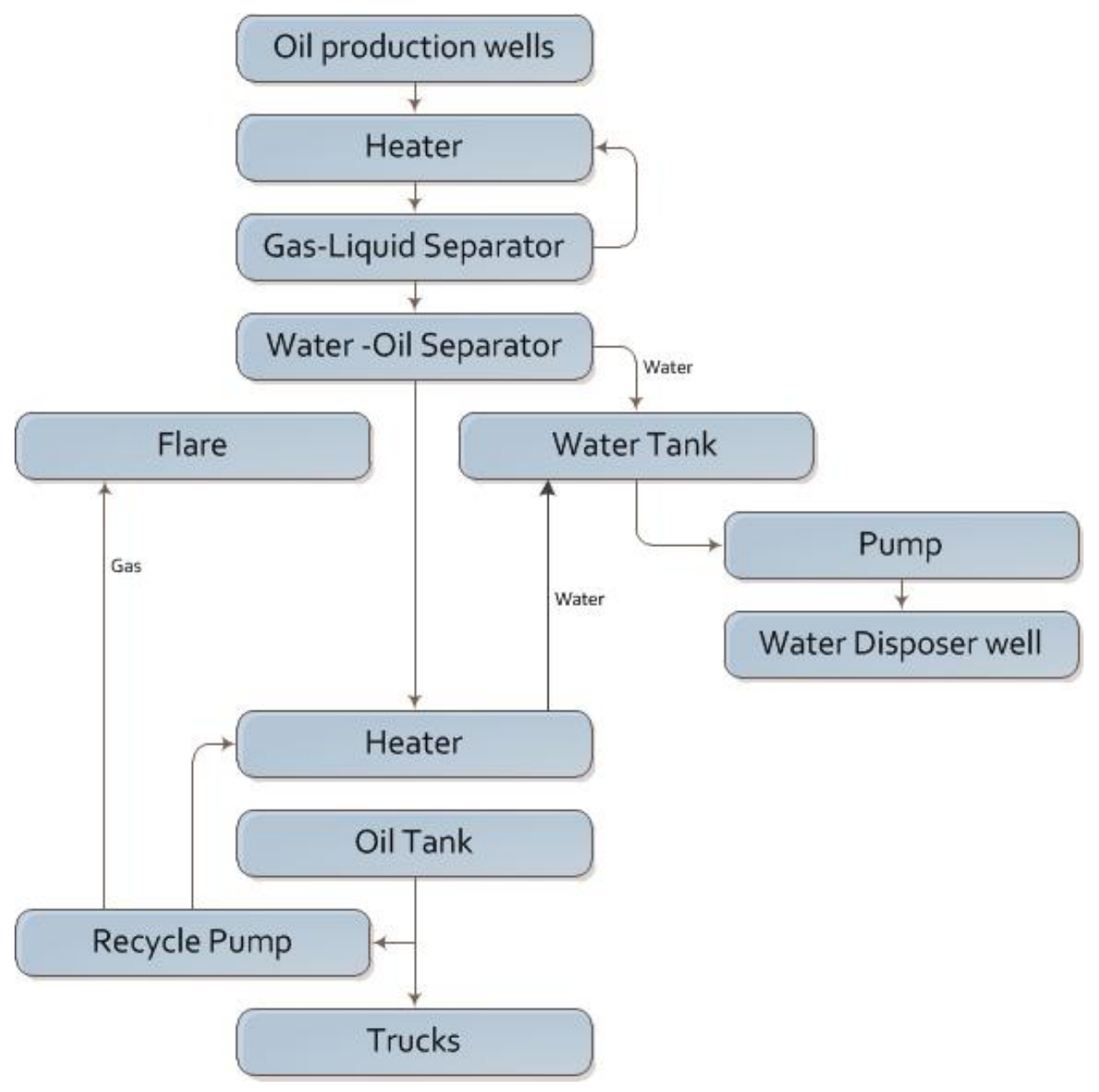

Figure 2. A schematic flow diagram of oil

Also, Operators of oil batteries often use personal experience as a basis for controlling process operations. However, due to inherent differences and uncertainties in operator experiences, optimal control of separation processes cannot be realized. When a crisis occurs, different operators may react differently and the performance can suffer. Hence, developing an automated controller can enhance consistency and efficiency of operations of the separation process, thereby improving quality of the output oil. Therefore, Liao et al. presented an automated fuzzy logic controller (FLC) that can be used to improve oil quality produced from a separation process [14].

\subsection{Food Produce}

Automation of industrial oven production lines used for cooking biscuits interests biscuit manufacturers both in France and Germany. For this control type, a conventional solution is not satisfactory due to the non-linearities, multiplicity and heterogeneity of sensitive parameters. Modelling of the cooking process is both complex and uncomplete. However, experienced operators are perfectly able to control cooking using their empirical knowledge. The main characteristics that can be measured in a biscuit are its colour, humidity and dimensions. These characteristics can be affected by variations in quality of pastry ingredients, environmental conditions and the time the biscuit remains in the oven... These influences must be compensated by oven setting and conveyor 
belt speed. Control of production quality of this kind of food process can be broken down into the following functional steps: a: conditioning and merging of data, b: evaluation of subjective quantities (linked to quality), c: diagnosis of quality deviations, d: decision-making, c subjective evaluation. Fuzzy logic enables qualitative variables to be taken into consideration and existing "professional" expertise to be used. Fuzzy rule bases have been used associated with other techniques [15]. (Table.1).

Table 1: functions and associated techniques

\begin{tabular}{|l|l|}
\hline Functions & Associated techniques \\
\hline Sensor melting & \\
\hline Subjective evaluation & Fuzzy classification \\
\hline Diagnosis & Fuzzy ladder \\
\hline Decision making Fuzzy rule bases & Fuzzy rule bases \\
\hline
\end{tabular}

\subsection{PH Controller}

Benz et. al. developed and tested a self adaptive computer based $\mathrm{pH}$ measurement and control system for the control of fermentation process in a laboratory reactor and for the neutralization of wastewater streams [16]. The controller was based on the fuzzy logic, which permitted the inclusion of subjective knowledge, often based on experience and not on a theoretical model. It also employed data and used primary knowledge (experimental results) directly. At the end of the study, the experiments showed that the fuzzy controller was able to adjust the $\mathrm{pH}$ value faster than the common PID controller.

\subsection{Reactor control}

In chemical engineering, chemical reactors are vessels designed to contain chemical reactions. Chemical engineers design reactors to maximize net present value for the given reaction.

\subsubsection{Bioreactor}

Penicillin G acylase (PGA) is an important enzyme used as biocatalyst in the production of semisynthetic b-lactam antibiotics. Many microorganisms produce this enzyme and recombinant Escherichia coli has been preferred use for industrial applications. Bacillus megaterium is one of the microorganisms that excretes this enzyme into the medium. As a consequence, separation and purification steps are simplified. On-line measurement of enzyme activity during cultivation using in-situ sensors is a difficult task in the industrial environment due to the lack of robust and inexpensive instrumentation. This work presents the results of a fuzzy logic algorithm used to determine the moment of maximum enzyme concentration during Bacillus megaterium cultivations in an aerated and stirred, automated lab-scale bioreactor. The fuzzy algorithm was written in FORTRAN, compiled as a dynamic link library and implemented on a platform developed in MSVisual Basic. Data were exchanged in real time between the platform and the supervisory system, which was coupled to the bioreactor. It was possible to determine the moment at which maximum enzyme activity was reached in several bioreactor assays. At this point, the end of the process was indicated to the operator. The results illustrate the importance of using reliable computational intelligence-based algorithms in biochemical reactions [17].

\subsubsection{Fluidized bed reactor}


Commercial gas-phase fluidized bed polyethylene reactors are operated in a relatively narrow temperature range between 75 and $1108{ }^{\circ} \mathrm{C}$. This is to ensure sufficient polymerization rates and maintain the temperature below the polymer melting point [18]. For this reason, tight temperature control is of utmost important. Proportional integral (PI) controllers are the most commonly used controllers in industrial applications because they are relatively easy to design, have simple control structure and inexpensive cost. However, they exhibit poor performance when exposed to unknown disturbances such as dead zones. A successful way of dealing with such nonlinearity is to use fuzzy logic, which features an improvement in the transient characteristic of the control performance. Fuzzy logic controllers based on the Takagi-Sugeno inference method applied for the regulation of the reaction temperature of the industrial bubbling fluidized bed reactors for polyethylene production. The simulation results showed that, the conventional fuzzy logic controller is able to prevent the offset [19].

\subsection{Batch crystallizer}

Batch and semi-batch crystallization processes are suitable for manufacturing the high value products such as special polymers, fine chemicals and pharmaceuticals. Sheikhzadeh et al. described a real time MIMO control system of a semi-batch, anti-solvent crystallization process for paracetamol in the mixture of two solvents. The performance of adaptive neuro-fuzzy logic controller was studied using unseeded and seeded crystallization. The controller effectively met the 'direct' objectives, which were to keep the super saturation and the difference between the numbers of chord length counts in the predetermined ranges, for both sets of experiments [20].

\subsection{Combustion process}

Combustion is a series of thermal. The process exhibits significant dynamical changes due to the characteristic of each combustion stage. To enable actions towards efficiency and lower emissions, real-time information about combustion quality is essential. The amount of carbon dioxide $\left(\mathrm{CO}_{2}\right)$ is one of the main quality measures in efficiency calculations. Carbon dioxide content in a flue gas is usually measured with different types of gas analysers. Operating costs and the lack of real-time performance may limit the usability of hardware analysers in continuous use. Ruusunen and Leivisk presented a fuzzy model-based approach for approximating carbon dioxide content in a wood combustion process. The model outputs are used to calculate real time combustion quality measures and lower caloric values of the wood fuel, including the approximation of the oxygen content [21]. 


\section{Conclusions}

Complex industrial processes are difficult to control because of inadequate knowledge of their behavior. This lack of knowledge is principally a lack of structural detail and it is this which prevents the use of conventional control theory. However, these processes are often controlled with great skill by a human operator who makes decisions on the basis of inexact and linguistic measures of the process state. Fuzzy logic is considered as a superset of standard logic which is extended to deal with the partial truth. It has become one of the most successful technologies for developing complex control systems. Fuzzy logic is a design methodology that can be used to solve real life problems. Chemical engineering has employed fuzzy logic in the piping risk assessment, safety analysis, batch crystallizer, combustion process, Food Produce, fluidized catalytic cracking Unit and separation Process. It has also been applied to process control (e. g. PH controller, furnace ...) and kinetics. Based on the results of this literature review, it found that in these filed high potential is exist for research. Also, further research should be conducted to found the other applications.

\section{References}

[1] Shoureshi, R. (1993). "Intelligent control systems: Are they for real?", Transactions of the ASME, 115, 392400.

[2] King, P. J., \& Mamdani, E. H. (1997), "The application of fuzzy control system to industrial processes". Automatica, 13(3), 235-242.

[3] Zadeh, L.A., (1971), "Similarity Relations and Fuzzy Ordering", Journal of Information Sciences, Vol. 3, pp. 177-206.

[4] Zadeh, L.A., (1965), “Fuzzy Sets”, Journal of Information Sciences, Vol. 8, pp. 338-353.

[5] Markowski, A.S., Mannan, M. S. "Fuzzy logic for piping risk assessment (pfLOPA)", Journal of Loss Prevention in the Process Industries 22 (2009) 921-927.

[6] Markowski, A., Sam Mannan, M. and Bigoszewska, A., "Fuzzy logic for process safety analysis", Journal of Loss Prevention in the Process Industries 22 (2009) 695-702.

[7] Dagdeviren, M., Yuksel, I, "Developing a fuzzy analytic hierarchy process (AHP) model for behavior-based safety management", Information Sciences 178 (2008) 1717-1733.

[8] Gurcanli, G. E., Mungen, U., "An occupational safety risk analysis method at construction sites using fuzzy sets" International Journal of Industrial Ergonomics, 39 (2009) 371-387.

[9] American Institute of Chemical Engineers, 3 Park Avenue, New York, NY 10016-5991, http:// www.aiche.org, Center for Chemical Process Safety, http://www.aiche.org/ccps/

[10] Osofisan, P.B., Obafaiye, O.J. "Fuzzy Logic Modeling of the Fluidized Catalytic Cracking Unit of a Petrochemical Refinery", The Pacific Journal of Science and Technology, Volume 8. Number 1. May (2007) 59-67.

[11] Taskin, H., Cemalettin, K., Uygun, $O$ and Arslankaya, S., "Fuzzy logic control of a fluid catalytic cracking unit (FCCU) to improve dynamic performance ", Computers and Chemical Engineering 30 (2006) 850-863.

[12] Tsekouras, G., Sarimveis, H., Raptis, C. and Bafas, G. "A fuzzy logic approach for the classification of product qualitative", Computers and Chemical Engineering 26 (2002) 429-438.

[13] Ghadimi, A., Sadrzadeh, M. and Mohammadi, T., "Prediction of ternary gas permeation through synthesized PDMS membranes by using Principal Component Analysis (PCA) and fuzzy logic (FL) ", Journal of Membrane Science 360 (2010) 509-521.

[14] Liao, R.F., Chan, C.W. , Hromek, J., Huang, G.H., and He, L., "Fuzzy logic control for a petroleum separation process", Engineering Applications of Artificial Intelligence 21 (2008) 835-845.

[15] Chevrie, F., Guely, F., " Fuzzy logic- Cahier technique no 191", first issued, december 1998.

[16] Benz, R., Menzl, S., Stühler, M., (1996), "A self adaptive computer based pH measurement and fuzzy control system", Wat. Res., Vol. 30, No. 4, pp. 981-991. 
M. R. Sarmasti Emami/ TJMCS Vol .1 No.4 (2010) 339-348

[17] E. R. Nucci; R. G. Silva; T. C. Gomes; R. C. Giordano; A. J. G. Cruz, "A fuzzy logic algorithm for identification of the harvesting threshold during PGA production by Bacillus", Brazilian Journal of Chemical Engineering, April 27, 2005.

[18] Xie, T., McAuley, K.B., Hsu, C.C. and Bacon, D.W., 1994, Gas phase ethylene polymerization: production processes, polymer properties, and reactor modeling, Ind Eng Chem Res, 33: 449-460.

[19] Ghasem, N. M., "Design of a fuzzy logic controller for regulating the temperature in industrial polyethylene fuidized bed reactor", Chemical Engineering Research and Design, 84(A2): 97-106.

[20] Sheikhzadeh, M., Trifkovic, M. and Rohani, S., "AdaptiveMIMOneuro-fuzzy logic control of a seeded and an unseeded anti-solvent semi-batch crystallizer", Chemical Engineering Science 63 (2008) 1261 - 1272.

[21] Ruusunen, M., Leivisk, K., "Fuzzy modelling of carbon dioxide in a burning process", Control Engineering Practice 12 (2004) 607-614. 